\title{
De la libre circulation au contrôle permanent
}

Les autorités françaises face aux mobilités tsiganes transfrontalières, 1860-1930

From Free Circulation to Permanent Control: the French Authorities face the Transborder Gypsy Mobility, 1860-1930

Ilsen About

\section{(2) OpenEdition}

Journals

Édition électronique

URL : http://journals.openedition.org/conflits/17757

DOI : $10.4000 /$ conflits. 17757

ISSN : $1777-5345$

Éditeur :

CCLS - Centre d'études sur les conflits lilberté et sécurité, L'Harmattan

Édition imprimée

Date de publication : 15 décembre 2009

Pagination : 15-38

ISBN : 978-2-296-11655-9

ISSN : 1157-996X

Référence électronique

Ilsen About, « De la libre circulation au contrôle permanent », Cultures \& Conflits [En ligne], 76 | hiver 2009, mis en ligne le 03 mai 2011, consulté le 30 mars 2021. URL : http://journals.openedition.org/ conflits/17757 ; DOI : https://doi.org/10.4000/conflits.17757 


\title{
De la libre circulation au contrôle permanent
}

\author{
Les autorités françaises face aux mobilités tsiganes \\ transfrontalières, 1860-1930
}

\section{Ilsen ABOUT}

Ilsen About est historien, docteur $d u$ European University Institute, assistant de recherches an CNRS entre 2008 et 2009 et actuellement chercheur post-doctorant à l'IRIS-EHESS. Spécialisé dans l'histoire des pratiques policières d'identification en France et en Italie entre 1880 et 1914, il est l'auteur, avec Vincent Denis, d'un ouvrage à paraître aux éditions La Découverte en 2010 qui présente une Histoire de l'identification des personnes. Ses recherches portent également sur la police des étrangers en France dans l'entre-deux-guerres, il a publié sur cette question "Identifier les étrangers. Genèses d'une police bureaucratique de l'immigration dans la France de l'entre-deux-guerres » dans Gérard Noiriel, dir., L'identification des personnes. Genèse d'un travail d'État, Paris, Belin, 2007. Ses recherches portent actuellement sur les politiques appliquées aux populations tsiganes en Europe de l'Ouest entre 1880 et 1939 et, en particulier, sur les mécanismes policier et administratif de contrôle et d'exclusion des minorités migrantes ou assimilées. Contact : ilsen.about@gmail.com; http://cnrs.academia.edu/IlsenAbout

E ntre 1860 et 1930, l'évolution des relations entre les agents de l'autorité et les groupes mobiles de «Bohémiens » ou « Romanichels », suivant les expressions employées à l'époque en France, montre combien les forces de l'ordre s'investissent alors dans le contrôle des populations tsiganes. L'assimilation progressive de ces communautés à un fléau social et la dénonciation des formes du vagabondage auxquelles elles sont abusivement associées, contribuent à rompre un équilibre, certes fragile, qui avait été établi depuis le XVIII e siècle avec les populations locales ${ }^{1}$. Un processus est alors enclenché qui conduit à la suppression des régimes d'échanges sociaux ou éco-

1. Pour une vue d'ensemble, cf. Fraser A., The Gypsies, Oxford, Blackwell, 1992 ; Asséo H., Les Tsiganes. Une destinée européenne, Paris, Gallimard, 1994 ; Piasere L., I rom d'Europa. Una storia moderna, Roma, Laterza, 2004 ; Zimmermann M. (dir.), Zwischen Erziehung und Vernichtung. Zigeunerpolitik und Zigeunerforschung im Europa des 20. Jabrhunderts, Stuttgart, Franz Steiner Verlag, 2007. 
nomiques constitués auparavant, et à la mise en œuvre d'une forme inédite de contrôle des personnes et de surveillance des déplacements. Ce processus historique complexe empruntant la voie d'une marginalisation sociale et d'une stigmatisation sur le plan du droit a été interprété comme l'expression d'une nationalisation impossible des Tsiganes en Europe entre le XIX et le XX siècle ${ }^{2}$. Cette « cassure » trouve notamment ses origines dans l'imposition d'un dispositif policier et administratif spécifique, particulièrement renforcé dans l'ensemble des pays d'Europe de l'Ouest entre la fin des années 1890 et la veille de la Première Guerre mondiale ${ }^{3}$.

Durant cette période, la question de la coopération policière, à l'échelle nationale ou internationale, semble avoir joué un rôle important dans l'élaboration globale des dispositifs de contrôle, même si les communications entre services de police, a fortiori divisés par les limites frontalières, demeurent largement embryonnaires. De premiers signes de collaboration existent pourtant déjà entre 1890 et 1914, mais il faut attendre le début des années 1920 pour voir apparaître un véritable cadre institutionnel chargé de coordonner les actions policières sur le plan international ${ }^{4}$. Il s'agira de montrer ici comment l'échange de données s'est imposé progressivement comme un élément capital des politiques de contrôle à l'égard des Tsiganes. En effet, cette préoccupation semble s'inscrire dans un processus interne aux forces de police, soucieuses de renforcer l'administration de la «mémoire policière ", en améliorant la saisie et la conservation des informations permettant d'identifier les personnes, et dans un processus qui stigmatise toujours davantage le libre franchissement des frontières entre départements et entre États.

Enregistrer les individus, s'assurer des documents d'identité en leur possession, surveiller les caravanes dans les campagnes ou dans les villes, empêcher le passage des bornes frontières, prévenir les délits et limiter les affrontements avec les populations locales, telles sont les tâches qui sont confiées aux agents de police, bien souvent débordés par l'ampleur de ces missions. À la fin du XIXe siècle, l'absence de véritable réglementation en la matière laisse encore

2. Cf. Asséo H., «L'invention des “nomades” en Europe au XXe siècle et la nationalisation impossible des Tsiganes ", in Noiriel G. (dir.), L'identification des personnes. Genèse d'un travail d'État, Paris, Belin, 2007, pp. 161-180.

3. Pour un parallèle avec l'Angleterre et l'Allemagne, cf. Behlmer G.K., “The Gypsy Problem in Victorian England”, Victorian Studies, vol. 28, n² 2, 1985, pp. 231-253 ; Mayall D., English Gypsies and State Policies, Hatfield, University of Hertfordshire Press, 1995 ; Bonillo M., "Zigeunerpolitik»im deutschen Kaiserreich, 1871-1918, Frankfurt am Main, Peter Lang, 2001.

4. Cf. Jensen R.B., " The International Anti-Anarchist Conference of 1898 and the Origins of Interpol”, Journal of Contemporary History, vol. 16, n² 2, 1998, pp. 323-347 ; López L., « Des gendarmes luxembourgeois chez les Brigades du Tigre : les prémices de la coopération policière transfrontalière en Europe occidentale », Revue de la Gendarmerie Nationale, $\mathrm{n}^{\circ} 226$, 2008, pp. 116-125. Pour une vue d'ensemble, voir, Deflem M., Policing World Society. Historical Foundations of International Police Cooperation, Oxford, Oxford University Press, 2002 ; Jäger J., Verfolgung durch Verwaltung. Internationales Verbrechen und internationale Polizeikooperation, 1880-1933, Konstanz, Universitätsverlag Konstanz, 2006. 
une très grande marge d'appréciation et les pratiques sur le terrain varient selon les circonstances, les lieux et les acteurs. Par la suite, tout l'enjeu de la modernisation des institutions de police en matière de surveillance des nomades semble résider dans la volonté d'améliorer le quadrillage du territoire, la collaboration entre forces de police et de gendarmerie, et, plus globalement, de renforcer la pression exercée sur ces populations considérées comme toujours plus dangereuses ${ }^{5}$. Le déroulement de situations concrètes de face-à-face entre les agents de l'autorité et des caravanes aux frontières et les effets immédiats de ces confrontations sur les transformations de la réglementation mobiliseront essentiellement l'attention afin de mettre en lumière les principales étapes du changement de conjoncture qui touche aux relations entre les autorités françaises et les populations tsiganes. En cela, ce texte constitue davantage une proposition empirique d'étude de ce phénomène par l'intersection de différents niveaux d'observation qu'une synthèse véritablement exhaustive. Ce parti pris n'a pas permis d'approfondir certaines questions, qui ne seront donc abordées que très indirectement, comme la position institutionnelle des différentes autorités de police impliquées et les relations entre elles, le travail concret des agents, le rôle de la presse, celui des relations diplomatiques, les réactions des populations tsiganes elles-mêmes face aux mesures de contrôle ${ }^{6}$.

En choisissant une perspective de longue durée, il s’agira donc plus généralement de confronter deux approches rarement mises en relation : d'une part, les transformations profondes de la police en France entre la fin $\mathrm{du}$ Second Empire et l'entre-deux-guerres, en termes à la fois institutionnel et professionnel, et d'autre part, les mutations radicales du régime européen de circulation, marquées par l'extension du monopole étatique des moyens légitimes de circulation ${ }^{7}$. C'est pour contribuer à la confrontation particulièrement fertile de ces deux perspectives qu'il s'agira donc ici d'articuler l'émergence d'une figure fantasmée du « Tsigane international », franchissant les frontières sans contrainte et fauteur de troubles en puissance, avec l'importance croissante attribuée à la centralisation de l'information policière et la circulation des données à l'intérieur des institutions de police, voire au-delà des frontières, à l'échelle internationale ${ }^{8}$.

5. Récemment Leo Lucassen a proposé d'analyser les projets d'emprise étatique sur les populations tsiganes et les difficultés rencontrées dans leur mise en ouvre comme un élément de critique du paradigme de la modernisation, employé pour décrire la transformation des États européens à l'époque contemporaine. Cf. Lucassen L., "Between Hobbes and Locke. Gypsies and the Limits of the Modernization Paradigm", Social History, vol. 33, n 4, 2008, pp. 423441.

6. Pour une vue d'ensemble sur les forces de police en France cf. Berlière J.-M., Le monde des polices en France, XIX ${ }^{e}-X X^{e}$ siècles, Bruxelles, Complexe, 1996 ; Blanc-Chaléard M.-C., Douki C., Dyonnet N., Milliot V. (dir.), Police et migrants en France, 1667-1939, Rennes, Presses Universitaires de Rennes, 2001 ; Berlière J.-M., Denys C., Kalifa D., Milliot V. (dir.), Métiers de police. Être policier en Europe, XVIII ${ }^{-} X X^{e}$ siècle, Rennes, Presses Universitaires de Rennes, 2008.

7. Cf. Torpey J., "Aller et venir : le monopole étatique des "moyens légitimes de circulation” », Cultures \& Conflits, $\mathrm{n}^{\circ}$ 31-32, 1998, pp. 63-100. 


\section{Des papiers sans qualité : les circulations tsiganes à la fin du XIXe siècle.}

A la fin du mois de mai 1866, un groupe de Tsiganes se présente dans la commune de Bettignies, située au Nord de la France à quelques centaines de mètres de la frontière belge 9 . Au premier jour de son arrivée, cette caravane est décrite comme une "bande de Bobémiens en guenilles au nombre de dix familles peut-être 60 à 80 personnes ayant 27 chevaux et autant de voitures $10 »$. Cette entrée massive, bruyante et spectaculaire est alors appréhendée comme une "irruption », et dès le premier jour, les autorités policières locales, représentées par le commissaire de la ville de Maubeuge, insistent sur la responsabilité de la gendarmerie belge dans le passage de la frontière ${ }^{11}$. Il semble en effet que ce groupe fut accompagné jusqu'au point de passage et, en quelque sorte, remis aux autorités françaises présentes sur place. Les conditions de leur passage et les moyens possibles de leur refoulement sont exposés en ces termes par le commissaire de Maubeuge :

«La douane à Bettignies les a retenus malgré le visa de leurs passeports par la légation de France. Ils doivent s'acquitter des droits d'entrée de leurs chevaux pour 800 francs; et malgré leur état de misère apparente, ils ont beaucoup d'argent. Je me transporte de suite à Bettignies pour les empêcher d'avancer plus loin [...]. D’après la réponse, je les ferai sortir, ou je les autoriserai à entrer en France 12 ».

Ces détails sont précieux. Ils indiquent d'une part que les chefs de la caravane possèdent des titres d'identités apparemment en règle, en l'occurrence des passeports munis de visas délivrés par les autorités consulaires françaises d'Allemagne. D'autre part, la possession de ressources financières, sur lesquelles pèsent des soupçons exprimés sans détour, témoigne d'une connaissance exacte des impératifs soumis au passage des frontières et, en particulier,

8. Pour un regard comparatif, cf. Hubert M.-C., " Les réglementations anti-tsiganes en France et en Allemagne avant et pendant l'occupation ", Revue d'histoire de la shoah, n 167, 1999, pp. 20-52 ; Asséo H., « La perception des Tsiganes en France et en Allemagne, 1870-1930 », in Gueslin A., Kalifa D. (dir.), Les exclus en Europe, 1830-1930, Paris, Editions de l'Atelier, 1999, pp. 221-233. Sur la figure du « Tsigane international », en Tchécoslovaquie durant l'entre-deux-guerres, voir Donert C., " Der "internationale Zigeuner" in der Tschechoslowakei. Eine transnationale Geschichte der Grenzkontrolle, 1918-1938 », in Duhamelle C., Kossert A., Struck B. (eds.), Grenzregionen. Ein europäischer Vergleich vom 18. bis 20. Jabrbundert, Frankfurt am Main, Campus Verlag, 2007, pp. 295-314.

9. Dossier «Bohémiens repoussés à la frontière : correspondance, 1866 », Archives départementales du Nord (AD Nord), M 204/10.

10. Dépêche télégraphique du commissaire de police de Maubeuge au Préfet du Nord, 29 mai 1866, AD Nord, M/204/10.

11. Sur la circulation des Tsiganes entre France et Belgique, cf. Coupain N., «L'expulsion des étrangers en Belgique (1830-1914)", Revue Belge d'Histoire Contemporaine, $\mathrm{n}^{\circ}$ 1-2, 2003, pp. 5-48; Nézer F., Évolution de la législation belge sur les étrangers indésirables de 1830 à 1914, mémoire de licence, Louvain-la-Neuve, Université catholique de Louvain, 2005.

12. Dépêche télégraphique du commissaire de police de Maubeuge au Préfet du Nord, 29 mai 1866, op. cit. 
des droits d'entrée obligatoires imposés sur les chevaux. Sans posséder apparemment de véritables moyens légaux pour empêcher leur entrée sur le territoire, le commissaire, soutenu par sa hiérarchie, prend cependant l'initiative de s'y opposer ${ }^{13}$. Il est important de souligner la très mauvaise réputation qui entoure la venue de cette caravane et la rumeur qui les accompagne : "d'après les bruits qui courent, ils volent, pillent dans les maisons où on ne leur oppose pas de résistance »; «depuis quelques jours on parlait d'une bande qui était renvoyée de chaque ville de la Belgique ${ }^{14} »$.

Les événements successifs offrent un exemple remarquable des stratégies de contournement alors déployées par les chefs de cette caravane face aux autorités et des conséquences de l'intervention autoritaire et peu préparée des forces de police sur la circulation des Tsiganes. Le passage au-delà du poste de douane entraîne l'installation d'un campement au centre même du village de Bettignies. Sur requête du commissaire de Maubeuge, les chefs de la caravane lui présentent des passeports en allemand portant des visas d'autorités étrangères, notamment un visa de l'ambassade de France à Berlin. S'exprimant en allemand, ils prétendent venir de Hongrie et affirment vouloir se rendre en Italie en traversant la France. Dans l'attente de consignes précises, le campement s'installe pour la nuit sous la surveillance du douanier et du garde-champêtre, non sans provoquer la curiosité et l'inquiétude des habitants du village. Le lendemain, l'ordre est reçu de procéder au refoulement de cette caravane et une scène de grande agitation se déroule alors sous les yeux du commissaire :

"Les hommes se mirent à vociférer, les femmes et les enfants à implorer le ciel, et il n'y eut qu'un seul moyen pour faire cesser ces démonstrations, ce fut de leur faire comprendre que les gendarmes venaient à cheval pour les conduire à la frontière. L'effet fut magique, les voitures furent attelées, et la bande, escortée par une dizaine de préposés armés et par le commissaire [...], prit la route de Belgique. Une fois le poteau frontière passé, ils se mirent à crier et à dire des grossièretés au commissaire [...] et s'enfuirent au plus vite au galop de leurs petits chevaux d'Ukraine 15 ».

Le départ précipité de cette caravane et la crainte de la voir revenir par une autre route traversant la frontière non loin de là entraînent l'envoi d'un télégramme destiné à avertir les autres postes frontières. Entre-temps la gendarmerie belge semble s'être alors « mise à leurs trousses pour les empêcher d'en-

13. Une dépêche de la préfecture est alors envoyée au commissaire pour lui préciser les instructions à suivre : «Ne laissez pas entrer sur le territoire français la bande de Bohémiens signalés par la mairie de Bettignies comme volant, pillant et commettant des attentats à la propriété ", Note manuscrite du Préfet du Nord, « Bohémiens repoussés à la frontière », 30 mai 1866, AD Nord, M 204/10.

14. Les italiques sont de nous. Citations tirées du rapport du commissaire de Maubeuge, 31 mai 1866, AD Nord, M 204/10.

15. Ibid. 
trer plus avant ", alors que, de leurs côtés, les agents français veillent toute la journée près de la frontière pour empêcher leur retour ${ }^{16}$. Quelques semaines plus tard, il apparaît cependant que cette même caravane est aperçue en Belgique à proximité de la frontière, puis en France même près de Maubeuge. Une enquête conduite dans les environs n'aboutit cependant à aucune certitude, tandis que des problèmes de communication entre les autorités traduisent l'impossibilité d'exercer un véritable contrôle dans les campagnes ${ }^{17}$.

L'intérêt de cet épisode est de mettre en lumière le caractère particulièrement empirique du contrôle de la mobilité frontalière en France à la fin du Second Empire. Les réformes de la police française à cette période et l'adaptation de l'appareil policier aux conditions du département du Nord avaient engendré précisément l'installation de nouveaux commissaires dans les villes de la frontière, comme Maubeuge, et la rapidité d'exécution du commissaire de cette ville à l'arrivée de la caravane témoigne d'ailleurs d'une certaine réactivité ${ }^{18}$. Mais les moyens mis à la disposition du commissaire local apparaissent très fragiles, comme l'atteste la menace imaginaire concernant l'intervention éventuelle des gendarmes. En réponse, les Tsiganes ont emmagasiné une expérience solide qui leur permet d'échapper à des interrogatoires trop poussés. L'usage de plusieurs langues, la présentation de documents d'identité et de visas inconnus des autorités, la possession d'argent en grande quantité, qui étonne, et peut attirer, selon les cas, la confiance ou la méfiance, constituent autant d'instruments mobilisés au service d'une stratégie faite de ruse, de stratagèmes subtils et d'une mise en scène de soi qui impressionne et trouble dans le même temps. Les Tsiganes mobiles qui parcourent les plaines de la frontière franco-belge bénéficient ainsi d'un cadre réglementaire très fragile ; leur circulation paraît certes gênée par l'intervention des autorités, mais la porosité des frontières et la connaissance très fine des territoires traversés leur permet finalement une très grande liberté, comme en témoignent de nombreuses études consacrées aux mobilités tsiganes en France au XIX siècle ${ }^{19}$.

Certes, dans les années 1860, plusieurs réglementations avaient tenté d'encadrer la circulation des populations mobiles en France, comme les textes de

16. Ibid.

17. Note du Commissaire de Maubeuge au Préfet du Nord, «Bohémiens repoussés de la frontière », 18 juin 1866, AD Nord, M/204/10.

18. Cf. Payne H.C., The Police State of Louis Napoléon Bonaparte, 1851-1860, Seattle, University of Washington Press, 1966 ; Caulier A., "S'adapter au temps et à l'espace. Le maillage policier dans le département du Nord sous le Second Empire », in Berlière J.-M., Denys C., Kalifa D., Milliot V. (dir.), Métiers de police. Etre policier en Europe, XVIII $I^{e} X X^{e}$ siècle, op. cit., 2008, pp. 333-344.

19. Sur la mobilité tsigane en France au XIXe et au début du XXe siècle, cf. Vaux de Foletier F. de, "Voyages et migrations des Tsiganes en France au XIXe siècle ", Études Tsiganes, n 3, 1973, pp. 1-30; Vaux de Foletier F. de, Les Bohémiens en France an XIXe siècle, Paris, Lattès, 1981 ; Reyniers A., "Pérégrinations des Jénis en France au XIXe siècle ", Études tsiganes, nº 2, 1991, pp. 19-25; Robert P., «La migration des Sinté piémontais en France au XXe siècle », Études tsiganes, $\mathrm{n}^{\circ} 18-19,2004$, pp. 29-51; Reyniers A., «Pérégrination des Manouches en France au $\mathrm{XIX}^{\mathrm{e}}$ siècle ", Études tsiganes, ${ }^{\circ}$ 26, 2006, pp. 9-17. 
principe instaurant officiellement le « carnet de saltimbanque » en 186320 . L'année suivante, la direction de la Sûreté publique du ministère de l'Intérieur déplorait explicitement l'absence de législation adaptée aux populations tsiganes ${ }^{21}$. Une circulaire prévoyait alors précisément de développer la surveillance de haute police, réservée habituellement aux criminels libérés, à tous les individus " connus sous le nom de Bohémiens » en leur qualité soit de vagabonds, soit d' " étrangers dangereux ». La direction de la Sûreté publique, dont la position à l'époque était particulièrement fragile et les moyens inexistants, ambitionnait de sédentariser les Tsiganes par une domiciliation forcée expliquée en ces termes :

« Le gouvernement pourra, en assignant à chacun d'eux une résidence distincte et obligée, les disséminer et rompre ainsi ces associations de malfaiteurs qui se livrent à la mendicité déguisée sous différentes formes, quand ils n'ont pas recours au maraudage ou au vol $22 »$.

L'absence de véritables moyens matériels et humains pour parvenir à mettre en ouvre une telle mesure condamne d'avance ce projet et l'appel à un renforcement des contrôles aux frontières reste, de la même manière, extrêmement théorique. Le défaut de centralisation en la matière pèse lourdement dans la possibilité d'appliquer une quelconque mesure de ce genre, alors même qu'une grande partie des décisions est déléguée aux préfets qui conservent alors une importante marge de manœuvre. Quelques années plus tard, à la fin de la guerre franco-allemande, une circulaire du ministère de l'Intérieur dresse d'ailleurs un constat pessimiste sur les mesures appliquées aux Tsiganes et traduit l'impuissance des autorités :

«On signale sur plusieurs points du territoire, la présence de Bohémiens dont les méfaits inquiètent les populations. L'administration est à peu près désarmée à leur égard : ils exercent un métier ou prétendent en exercer un, et par conséquent ne peuvent être condamnés comme vagabonds, d'ailleurs l'autorité ne peut plus exercer à leur égard une surveillance efficace puisque, depuis le décret du 8 décembre 1851, les individus placés sous la surveillance de la haute police choisissent librement leur résidence et qu'on ne peut de ce fait disperser les bandes en des localités diverses ; enfin, on ne peut les expulser, les gouvernements étrangers ne voulant jamais les accepter. Il importe absolument d'empêcher de pénétrer

20. Circulaire de la direction générale de la Sûreté publique, 6 janvier 1863, Archives nationales, Centre des archives contemporaines (AN CAC 19940494/111), dossier 6270. Sur ce sujet, cf. Wagniart J.-F., Le vagabond à la fin du XIXe siècle, Paris, Belin, 1999.

21 . Circulaire de la direction générale de la Sûreté publique, «Au sujet des étrangers connus sous la dénomination de Bohémiens », 19 novembre 1864, AN CAC 19940494/111, dossier 6270.

22. Ibid. 
sur le territoire national tous les individus qui ne pourraient justifier de leur identité et de leur nationalité 23 ».

Les préfets sont donc appelés à repousser vigoureusement tous les individus qui ne seraient pas à même de justifier ces deux éléments lors du passage des frontières, les limites du territoire étant dès lors envisagées comme un rempart face aux éventuelles migrations de Tsiganes vers l'intérieur du pays. Ces propositions traduisent une nouvelle fois l'inefficacité globale de toutes les mesures en vigueur et l'appel réitéré à une surveillance accrue des frontières apparaît comme un signe supplémentaire d'impuissance. Entre 1872 et 1895, plusieurs circulaires spéciales sont transmises aux préfets et les agents sur le terrain sont appelés régulièrement à « un redoublement de vigilance et de sévérité » ou à interdire le stationnement de caravanes dans certains départements 24 .

La première mesure d'envergure concernant le contrôle des Tsiganes a lieu en mars 1895 sous la forme du dénombrement effectué à l'échelle nationale concernant tous les «nomades, bohémiens et vagabonds » circulant en France. Plusieurs épisodes de violence envers les Tsiganes à la fin XIX ${ }^{\mathrm{e}}$ siècle, une « intolérance administrative » de plus en plus grande dans les départements et la pression exercée par les élus locaux sur la représentation nationale constituent alors les éléments principaux d'un contexte, souvent étudié dans une perspective de longue durée, comme un moment important de transition vers un régime plus sévère de contrôle ${ }^{25}$. Pour les contemporains, l'opération de recensement de 1895 est alors perçue comme un remède infaillible, comme en témoigne un article du Petit journal:

«Le même jour, à la même heure, partout en France, ils ont été cernés par la gendarmerie; il leur a fallu dire leurs noms, prénoms et lieux d'origine, de sorte que maintenant, il sera possible de les soumettre aux lois qui régissent les étrangers en France 26 ».

Effectué dans la seule journée du 20 mars 1895, ce recensement demeure toutefois partiel et lacunaire car les gendarmes et les gardes-champêtres, chargés de l'opération, ne parviennent pas à couvrir l'étendue de chaque département. Les registres composés pour l'occasion rassemblent des informations

23. Circulaire de la Sûreté générale, 4 avril 1872, AN CAC 19940494/111, dossier 6270.

24 . Circulaire de la direction de la Sûreté publique, 26 mai 1874 ; Arrêté du Préfet de Saône-etLoire, 20 mars 1874 ; circulaire du 5 janvier 1881 ; circulaire du 29 juin 1889, AN CAC 19940494/111, dossier 6270.

25. Cf. Filhol E., «Les Tsiganes en France : du contrôle à la répression (1895-1946) », Revue trimestrielle des droits de l'Homme, $\mathrm{n}^{\circ}$ 68, 2006, pp. 989-1008; Asséo H., « Pourquoi tant de haine ? L'intolérance administrative à l'égard des Tsiganes de la fin du XIXe siècle à la veille de la deuxième guerre mondiale ", Diasporas, Histoire et Sociétés, n 10, 2007, pp. 50-67 ; Sitou E., «L'affaire des Gitanos : chronique d'une flambée raciste à Toulouse à la fin du XIXe siècle ", Études tsiganes, $\mathrm{n}^{\circ} 30,2008$, pp. 10-25.

26 . «Les camps-volants : recensement des Bohémiens en France », Le Petit Journal, 5 mai 1895. 
permettant d'identifier et de suivre les individus et les groupes, mais les possibilités d'homonymies, d'usurpations ou de dissimulation d'identité demeurent encore bien réelles. Des documents d'identité, délivrés par les mairies de résidence et réalisés pour l'occasion, comportent les noms et prénoms des membres de chaque "bande », la "profession apparente », la nationalité, le sexe et l'âge, le lieu de naissance, le lieu d'où ils viennent et celui où ils disent se rendre. Cependant aucun système d'enregistrement centralisé des documents délivrés n'est alors envisagé. Une commission spéciale extraparlementaire examine les résultats de ce recensement et produit, en mars 1898, un rapport qui souligne l'importance numérique de la population nomade, évaluée à 25000 individus, et l'insuffisance des personnels de police susceptibles de les surveiller 27. L'absence de formation adéquate, voire l'incompétence des gardes-champêtres est l'objet des critiques les plus vives, mais c'est plus généralement la faiblesse de la police des campagnes qui est soulignée. La création d'un « contrôle permanent de tous les agents chargés de la police des campagnes » est ensuite proposée par le ministère de l'Intérieur, en juin 1898. Dans les années 1900, la pression à l'encontre des nomades, et des nomades étrangers en particulier, s'accroît d'autant plus que le principe d'identification se diffuse au sein de la police française ${ }^{28}$. Pour autant, aucune mesure nationale globale n'est prise, tandis que les séries de règlements adoptés par les préfectures tentent d'apporter localement des réponses pour contrôler ces populations ${ }^{29}$.

La multiplication des cas impliquant des caravanes de Tsiganes franchissant les frontières entre la Suisse, l'Italie, la Belgique ou l'Allemagne, dans les premières années du $\mathrm{XX}^{\mathrm{e}}$ siècle, place les autorités de police face à leur impréparation et influence directement la transformation d'une question essentiellement locale en question politique, traitée au plus haut niveau de l'État. L'amélioration de la coopération policière et l'entente internationale sur ce qui est désormais nommé la « question tsigane » s'affirme alors comme l'objectif central des réformes à venir.

27. Cette commission instituée par décret le 13 novembre 1897 se nomme exactement Commission chargée de rechercher les moyens propres à assurer une surveillance plus étroite des vagabonds et gens sans aveu et à faciliter la découverte des auteurs de crimes et de délits. Cf. Challier F., La nonvelle loi sur la circulation des nomades. Loi du 16 juillet 1912, Paris, Librairie de jurisprudence, 1913, p. 152. Pour l'ensemble de cette période cf. Delclitte C., Nomades et nomadisme. Le cas de la France, 1895-1912, mémoire de maîtrise, Paris, Université Paris 8, 1995.

28. Cf. About I., "Les fondations d'un système national d'identification policière en France (1893-1914). Anthropométrie, signalements et fichiers », Genèses, n 54, 2004, pp. 28-52.

29. Cf. les éléments de rappel sur les années 1900 dans Filhol E., Un camp de concentration français. Les Tsiganes alsaciens-lorrains à Crest, 1915-1919, Grenoble, Presses Universitaires de Grenoble, 2004. 
Police des nomades et projet de coopération internationale dans les années 1900

Au début du siècle, le régime de circulation des Tsiganes en Europe semble subir un basculement brutal. L'exacerbation des sentiments xénophobes et la consolidation d'une conception ethnique de la nation libèrent la parole publique. L'expression du sentiment anti-tsigane se manifeste alors de plus en plus ouvertement, ce dont témoignent un grand nombre d'articles de presse qui usent et abusent de métaphores jouant sur l'idée de menace : «invasion », « déferlement », « irruption », " fléau », tels sont les termes qui accompagnent l'évocation des Tsiganes dans les journaux. Cette conjoncture est marquée par une contagion des discours, pour reprendre l'expression de Laurent Dornel 30 : le rejet du vagabondage et la condamnation de l'errance cö̈ncident avec la stigmatisation de la pauvreté et la dénonciation de l'étranger. Une «science du nomadisme " décrit les caractères anthropologiques et les pathologies criminelles d'un groupe désigné à la fois comme allogène sur le plan national et ethnique et inadapté socialement ${ }^{31}$. L'histoire d'une caravane expulsée de Suisse vers la France en 1907 reflète cette radicalisation des sentiments anti-tsiganes et éclaire les conditions dans lesquelles se développe un système policier et administratif de contrôle destiné à circonscrire la mobilité tsigane transfrontalière qui finalement s'applique, dès les années 1910, à un bien plus grand nombre.

Par sa situation géographique, la Suisse abritait périodiquement de nombreuses caravanes migrantes qui transitaient à travers le pays pour rejoindre l'Allemagne, la France ou l'Italie ${ }^{32}$. Déjouant la surveillance des autorités de police, fractionnées en brigades de gendarmerie dont la juridiction se limitait aux frontières des cantons, les caravanes circulaient alors assez librement jusqu'au début du XX $\mathrm{X}^{\mathrm{e}}$ siècle. En 1906, les signalements des autorités municipales et cantonales s'accumulent et une série de décisions est prise par le gouvernement fédéral ${ }^{33}$. Deux décrets imposent d'une part l'interdiction de traverser les frontières à toute personne reconnue comme tsigane et, d'autre part, la défense d'utiliser le système ferroviaire du pays. Ces décrets ont pour conséquence de légitimer la méfiance envers les Tsiganes et exacerbent une animosité qui se transforme parfois en violence. Peu de temps après, en novembre 1906, plusieurs affrontements opposent une troupe de Tsiganes à

30. Dornel L., La France hostile. Socio-histoire de la xénophobie (1870-1914), Paris, Hachette, 2004, p. 255.

31. Pour une vue générale sur l'histoire des sentiments anti-tsiganes, cf. Uerlings H., Patrut I.-K. (dir.), Zigeuner und Nation: Repräsentation - Inklusion - Exklusion, Frankfurt am Main, Peter Lang, 2008.

32. Cf. Huonker T., " "Jusqu'à la ceinture dans le grand marais". Roma, Sinti et Yéniches en Suisse, quelques aspects d'une persécution de longue durée ", Le cartable de Clio, n 4, 2004, pp. 130-136; Huonker T., Ludi R., Roms, Sintis et Yéniches. La "politique tsigane » suisse à l'époque du national-socialisme, Lausanne, Éditions Page deux, 2009.

33. Cf. Egger F., "Der Bundesstaat und die fremden Zigeuner in der Zeit von 1848 bis 1914 », Studien und Quellen, n 8, 1982, pp. 49-74. 
des habitants d'un petit village du canton de Berne : une battue est organisée par la population locale, des patrouilles de police et de pompiers sillonnent le territoire, des gendarmes sont ensuite dépêchés sur les lieux et conduisent l'ensemble de la caravane à la prison de Fribourg ${ }^{34}$. Dès lors, l'hostilité publique et la ténacité des agents de police provoquent l'expulsion répétée des caravanes d'un lieu à l'autre. Le regard porté sur ces communautés est alors résumé en ces termes par le Journal de Genève :

"Les gens que le peuple appelle "Bohémiens" sont de nouveau légion dans nos forêts. Si ces gens vivent à l'air du temps, ils n'en vivent pas. N'ayant rien semé, ils récoltent quand même et les déprédations qu'ils commettent sont innombrables [...]. Les polices cantonales jouent à la balle avec ces gens, témoin la caravane qui passa la nuit sur un pont l'année dernière, parce que, à chaque bout du pont, il y avait des gendarmes de deux cantons différents, qui empêchaient l'invasion de leur territoire 35 ».

Cette image d'une caravane immobilisée au milieu d'un pont est la métaphore visuelle d'un non-lieu juridique et politique dans lequel sont confinés, de plus en plus ouvertement, les Tsiganes. Si la dissimulation restait encore possible quelques années auparavant, les expulsions successives provoquent une visibilité de plus en plus grande, amplifiée par la couverture qu'en donnent les journaux. Ces migrations forcées engagent les autorités à coordonner de plus en plus sérieusement l'action policière. En mars 1907, une conférence inter cantonale est donc organisée entre les cantons de Vaud, de Fribourg et de Berne ${ }^{36}$. Il est établi que chaque canton doit parvenir à contenir les groupes qui circulent sur leur territoire sur la base d'un enregistrement des caravanes. En outre, l'enfermement d'une partie des Tsiganes à la prison de Fribourg est également l'une des décisions prises lors de cette conférence. Mais il s'agit alors moins d'une répartition des Tsiganes sur le territoire et d'une déclaration de principe que d'un véritable plan de coordination de l'action policière.

En juillet 1907, une caravane tsigane est immobilisée à la frontière entre la France et la Suisse, non loin de Genève ${ }^{37}$. Ayant obtenu un laissez-passer délivré par le consul allemand de cette ville, ils se dirigent vers la France pour officiellement rejoindre l'Allemagne par les Vosges. Ils sont alors interceptés par la gendarmerie qui décide de les raccompagner vers la frontière suisse, au

34. Cf. "Les Tziganes ", Journal de Genève, 23 novembre 1906 ; "Fribourg - La tribu des Tziganes ", Journal de Genève, 24 novembre 1906.

35 . «Encore les Tziganes », Journal de Genève, 25 novembre 1906.

36 . «Les Tziganes », Journal de Genève, 24 mars 1907.

37. Sur cet épisode, cf. «Les Bohémiens à la frontière ", Journal de Genève, 4 juillet 1907, 5 juillet 1907, 21 juillet 1907, 27 juillet 1907 ; « Les Bohémiens quittent Moillesulaz », Journal de Genève, 25 juillet 1907 ; "Les Romanichels de Moillesulaz », Journal de Genève, 11 août 1907, 16 août 1907, 22 septembre 1907 ; « Nos Romanichels ", Journal de Genève, 27 septembre 1907. 
pont de Moillesullaz. Celle localité était un lieu habituel de passage des Tsiganes, la mémoire d'un stationnement prolongé en 1899 et l'épidémie de petite vérole qui s'était déclarée à ce moment-là ayant marqué les esprits de la population locale. Au moment du passage, un concours de circonstances provoque une confrontation directe entre les forces de l'ordre des deux pays :

«Pendant que l'on examinait leurs titres, les trois chariots s'étaient engagés sur le pont. Comme il n'y avait pas moyen de leur faire rebrousser chemin sur le pont même, et qu'au surplus une automobile était survenue, qui fermait la colonne, on leur fit dépasser le pont. Mais à peine la frontière était-elle franchie que les gendarmes français s'y installèrent : "vous les avez, gardez les !” 38 ».

Dans ce climat houleux, des renforts sont demandés de part et d'autre et la caravane se trouve littéralement encerclée par des douaniers et des gendarmes. Un campement est alors installé non loin du pont, du côté suisse, et y demeure pendant plusieurs semaines. Immédiatement, les Tsiganes vendent leurs chevaux aux paysans des environs. Cette vente avait-elle pour but d'obtenir rapidement une somme d'argent importante ou l'immobilisation physique de la caravane était-elle plutôt destinée à décourager les forces de l'ordre de les expulser d'un côté ou de l'autre de la frontière ? Il n'est pas exclu que la vente des chevaux ait constitué un instrument traditionnel de diversion dans ce genre de cas, la nécessité de l'expulsion favorisant par la suite l'acquisition de chevaux à bas prix ${ }^{39}$.

En une journée, la caravane s'agrandit par l'arrivée de plusieurs familles provenant de France : «c'est une véritable invasion renouvelée des Hans » annonce dramatiquement le Journal de Genève ${ }^{40}$. Un recensement est pratiqué par les autorités suisses et des tractations diplomatiques sont engagées entre les deux pays pour parvenir à une décision, tandis qu'un journaliste dresse le tableau du campement : à la fois fasciné et méfiant face aux modes de vie des Tsiganes, il témoigne du climat de tensions qui entoure les roulottes ${ }^{41}$. Trois semaines plus tard, le ministère de l'Intérieur français autorise la traversée de l'est du pays vers l'Allemagne suivant un itinéraire décidé préalablement et sous l'escorte des gendarmes. Dans le Jura, ils sont interceptés par la brigade de gendarmerie de Salins-les-Bains qui procède à l'identification de tous les membres de la caravane et communique les fiches signalétiques au Service de l'identité judiciaire parisien. La reconnaissance de plusieurs Tsiganes mène à leur placement en détention pour des délits commis auparavant et la révélation des différentes mesures d'expulsion prononcées contre

38 . «Les Bohémiens à la frontière ", Journal de Genève, 4 juillet 1907.

39. Cf. à ce sujet Vaux de Foletier F. de, "La civilisation du cheval dans le monde tsigane ", Études tsiganes, $\mathrm{n}^{\circ}$ 28, 1982, pp. 1-19.

40. «Les Bohémiens à la frontière », Journal de Genève, 5 juillet 1907.

41 . «Merlifiches. Croquis », Journal de Genève, 8 juillet 1907. 
certains d'entre eux tend à accélérer la reconduite vers la frontière. Autour de Belfort, l'évacuation de cette caravane se heurte toutefois aux gendarmes allemands qui ont été prévenus de cette arrivée et s'y opposent. Au cours du mois d'août 1907, plusieurs allées et venues d'un côté à l'autre de la frontière contraignent les autorités françaises à adopter un autre itinéraire, vers la Suisse de nouveau. Enfin la caravane se heurte à nouveau aux autorités suisses et doit rebrousser chemin vers la France, dans le département du Doubs où leur trace se perd, sans doute temporairement.

L'histoire de cette caravane n'est pas isolée et plusieurs cas similaires peuvent être mentionnés pour la même période. L'exemple à la fois le plus étonnant et le plus extrême est rapporté par le journal Le Temps du 4 février $1908{ }^{42}$. Une roulotte transportant une famille de huit personnes fut immobilisée à la frontière entre la Belgique et la France à partir d'octobre 1907 et pour près de cinq mois à l'emplacement même du poteau indiquant la limite entre les deux pays. De chaque côté, des gendarmes exercèrent une surveillance permanente qui entraîna l'installation de guérites construites spécialement. Le coût de cette opération s'élève de jour en jour, le personnel qui se relaie et le temps passé à l'exercice de cette tâche entraînent la recherche d'une solution et l'abandon du dispositif 43. Entre 1907 et 1908, à la frontière entre la Suisse et l'Italie, ce sont plusieurs caravanes qui sont expulsées à plusieurs reprises vers l'un ou l'autre pays ; plusieurs affaires retentissantes entraînent alors une surveillance spéciale des routes et des transports ferroviaires ${ }^{44}$.

C'est précisément dans ce contexte qui voit se multiplier les confrontations directes entre forces de l'ordre et caravanes tsiganes que la Suisse prend l'initiative de réunir, au début de l'année 1908, une « conférence internationale à organiser en vue de règlement sur la question tsigane 45 ». Le projet prévoit l'obligation d'un contrôle d'identité individuel, la délivrance d'un certificat intérimaire portant les mensurations anthropométriques, la création de fichiers spécialisés et d'offices centraux nationaux reliés entre eux par un «office central d'enregistrement anthropométrique de tsiganes 46 ». En clair, cette proposition prévoit d'unifier à l'échelle européenne la communication des données personnelles concernant tous les Tsiganes circulant dans l'ensem-

42. « Histoire de Romanichels », Le Temps, 4 février 1908.

43. D’autres cas similaires sont évoqués pour la même période dans Arsac H., La loi du 16 juillet 1912 sur l'exercice des professions ambulantes et la réglementation de la circulation des nomades, ses causes, ses précédents, sa portée et son application pratique, Thèse de droit, Lyon, Bosc frères, M. et L. Riou, 1933.

44. Archivio Centrale delle Stato (ACS), PS, div. polizia, polizia giudiz. 1907-1909, b. 92, Note du 21 septembre 1908, Légation italienne à Berne à PS (Rome), « Trasporto di Zingari »; ACS, PS, div. polizia, polizia giudiz. 1907-1909, b. 92, Note du 3 juillet 1908, Préfecture de Côme à PS (Rome), « Carovane di nomadi guidate da Lehmann ».

45. «Pour les Tsiganes », Journal de Genève, 19 janvier 1908.

46. ACS, PS, div. polizia, polizia giudiz. 1910-1912, b. 354, « Projet d'un programme pouvant servir de base aux délibérations d'une conférence internationale à organiser en vue d'un règlement de la question tzigane », 9 p. 
ble des pays signataires d'un futur accord. Très explicitement, ce projet souhaite répondre aux passages illégaux de frontières et suppose que « l'État sur le territoire duquel ont été trouvés les tsiganes [fasse] les démarches nécessaires pour que ces nomades soient reconnus par leur pays d'origine ou soient naturalisés ou réintégrés dans leur nationalité », une commission internationale étant envisagée pour régler les cas litigieux ${ }^{47}$. Devant cette proposition, la direction de la police italienne soutient que la législation existante sur les Tsiganes et les dispositions prises à l'encontre des « étrangers dangereux pour l'ordre public » sont suffisantes et estime que l'initiative suisse porterait atteinte à la liberté d'action des autorités de police. Par ailleurs, le ministère de l'Intérieur italien considère qu'il n'existe aucune « classe de personnes de nationalité italienne, qu'il pourrait être possible de considérer comme appartenant à la catégorie des Tsiganes véritables ", populations qui habiteraient uniquement dans certains états balkaniques et d'Europe centrale ${ }^{48}$. Suivant une rhétorique paradoxale, la négation de l'existence même des Tsiganes en Italie voisine avec la volonté d'éviter toute ingérence extérieure dans l'action de la police à l'égard de ces mêmes populations. Les autres pays voisins de la Suisse rejoignent ce point de vue et refusent de "nationaliser » ces populations dénoncées comme étrangères, condamnant par là même le projet de conférence helvétique. Le refus de partager le champ des prérogatives nationales relevant des activités de police et la méfiance des hautes instances policières dans le partage des informations contribuent également à l'échec de cette proposition.

L'horizon voilé d'un cadre international de coopération policière n'écarte pas cependant le renforcement des réglementations sur le plan national, inspiré par les mesures générales prises en Allemagne entre 1907 et $1911{ }^{49}$. Précisément, l'affaire de la caravane de Moillesulaz suscite en France l'intervention de Ferdinand David, député de Haute-Savoie, au Parlement français et la rédaction d'une interpellation officielle du Président du Conseil concernant les «incursions de romanichels » 50. Déposé à l'automne 1907, ce texte est le premier d'une série qui forme le terrain de discussion préparant le vote de la loi de 1912 sur la circulation des «nomades» (Tsiganes) et forains en France ${ }^{51}$. Cette loi marquante qui impose à tout Tsigane résidant en France le port d'un carnet anthropométrique, créé sur le modèle des documents employés depuis les années 1880 pour identifier les criminels récidivistes libé-

\section{Ibid.}

48 . ACS, PS, div. polizia, polizia giudiz. 1910-1912, b. 354, Courrier du 20 août 1909, PS (Rome) à Ministère des Affaires étrangères, "Zingari - Conferenza internazionale proposta dal governo elvetico ".

49. Cf. Bonillo M., “Zigeunerpolitik”..., op. cit.

50 . «À la chambre française - Interpellation de David : les Romanichels ", Journal de Genève, 30 octobre 1907 ; "Sur la route ", Le Petit Parisien, 3 août 1907. Cf. Fernand David, Interpellation sur les mesures que compte prendre M. le Président du Conseil, ministre de l'Intérieur, pour assurer la sécurité dans nos campagnes et mettre fin aux incursions de Romanichels qui infestent notre territoire, J.O., Chambre des députés, séance du 29 octobre 1907. 
rés, doit être placé dans le contexte plus large des mesures prises à la même période en Europe de l'Ouest. La Suisse instaure par exemple en 1913 un système similaire d'enregistrement, dit "Zigeunerregistratur ", conçu comme la première marche vers une expulsion de toutes les caravanes vers l'étranger.

Entre 1907 et 1914, la pression policière exercée sur les migrations tsiganes change de nature ${ }^{52}$. Il ne s'agit plus de contenir ou de refouler suivant les cas, mais d'obtenir l'assurance d'une expulsion rapide et définitive des caravanes qui transitent par les zones frontalières. Le rejet des «nomades ", qui est alors admis par une classe politique majoritairement conservatrice comme un enjeu commun, correspond à l'adoption de méthodes visant à identifier les individus d'une part et permettre le contrôle de la circulation d'autre part. Le premier objectif, comblé par l'emploi des nouvelles méthodes de l'identification judiciaire, doit permettre d'individualiser le contrôle et de savoir avec certitude si tel ou tel individu a déjà été condamné ou expulsé afin de pouvoir prononcer des mesures judiciaires adaptées en cas de nouvelle arrestation et de légitimer, le cas échéant, un refoulement vers la frontière. Le second objectif est rempli en théorie par l'usage de documents portatifs, comme le carnet anthropométrique, qui conservent les traces du parcours suivi par les individus afin de pouvoir définir la réalité de la circulation sur le territoire national. Cet objectif est aussi atteint grâce à l'usage encadré de documents spéciaux, fiches d'information, rapports, listes, qui permettent d'améliorer la communication des informations entre les services de police sur le terrain et avec les services centraux installés au ministère de l'Intérieur, qui connaissent partout en Europe, entre 1900 et 1914, une phase importante de modernisation. Le contrôle des Tsiganes peut même être analysé comme la raison d'être de l'action d'une nouvelle « police du territoire » incarnée par des forces mobiles censées marquer l'avènement d'une « police nationale » 53 .

Le corollaire de cette phase de renforcement du contrôle des Tsiganes est le développement de techniques propres de contournement : changement des moyens de transports par la vente ou l'achat de roulottes ou de chevaux, stra-

51. Sur la loi de 1912, cf. Delclitte C., Nomades et nomadisme..., op. cit. ; Delclitte C., « La catégorie “nomade” dans la loi de 1912 », Hommes E migrations, 1995, pp. 23-30; Piazza P., «Au cœur de la construction de l'État moderne. Socio-genèse du carnet anthropométrique des nomades ", Les Cabiers de la sécurité, n 48, 2002, pp. 207-227 ; Filhol E., "La loi de 1912 sur la circulation des "nomades" (Tsiganes) en France ", Revue européenne des migrations internationales, vol. $23, \mathrm{n}^{\circ} 2,2007$, pp. 135-158 ; Filhol E., « Une mobilité sous haute surveillance : la loi de 1912 relative à la circulation des "nomades" (Tsiganes) en France ", Revue drômoise, vol. $98, \mathrm{n}^{\circ}$ 529, 2008, pp. 20-38.

52 . Asséo H., « La gendarmerie et l'identification des «nomades» (1870-1914) », in Luc J.-N. (dir.), Gendarmerie, État et Société au XIXe siècle, Paris, Publications de la Sorbonne, 2002, pp. 301-311 ; Berlière J.-M., " "Armer les pouvoirs publics contre un fléau social” ? La république et les nomades (1880-1914) ", Études tsiganes, 2004, pp. 52-64. Pour un parallèle avec l'Allemagne, cf. Lucassen L., " "Harmful tramps". Police Professionalization and Gypsies in Germany, 1700-1945 ", Crime, Histoire E Sociétés, vol. 1, n 1, 1997, pp. 29-50.

53. López L., "Les archives contre la statistique officielle ? Retour sur les brigades du Tigre (Dijon, 1908-1914) », Genèses, n 71, 2008, pp. 106-122. 
tégies dilatoires face aux forces de l'ordre et développement d'une rhétorique particulière conduisant à la confusion (mélange des langues, imprécision concernant les origines, diversité des documents d'identité produits), usurpation d'identité et usage de plusieurs patronymes, prénoms ou surnoms, destruction des documents d'identité. Globalement la pression policière aux frontières et le nouveau cadre réglementaire qui entoure les Tsiganes conduisent ainsi à un passage vers un régime de l'illégalité permanente qui les place constamment non seulement à la lisière, physique et symbolique, des États mais aussi à la marge du droit.

\section{Les Tsiganes pris au piège : la fermeture des frontières dans les années 1920}

Durant la Première Guerre mondiale, la création en France de plusieurs camps de rétention destinés aux Tsiganes semble marquer un durcissement temporaire des mesures de contrôle ${ }^{54}$. Cependant, cette solution répressive ne concerne qu'une très petite minorité d'individus et disparaît à la fin du conflit. La majeure partie des Tsiganes sur le reste du territoire connait une limitation des possibilités de circulation, tout comme le reste de la population, et bénéficie apparemment d'un relâchement de la pression policière. Une circulaire de 1920 évoque en effet «la suppression, depuis le début des hostilités, des tournées périodiques organisées [...] par la police mobile » qui réalisaient les signalements anthropométriques et les photographies judiciaires ${ }^{55}$. Suivant ce document, il apparaît donc que dans un certain nombre de départements, aucun carnet anthropométrique ne fut délivré durant la Première Guerre mondiale. Cette parenthèse, cependant, ne dure pas.

Après 1918, le contrôle effectif des nomades sur le territoire français se réorganise progressivement ${ }^{56}$. L'imposition du carnet entraîne tout d'abord l'interception des individus sur le territoire et leur immobilisation temporaire. Les personnes dépourvues de carnet sont conduites de force par les agents de l'autorité, policiers ou gendarmes, vers des services spécialement équipés ou des installations provisoires établies pour l'occasion, comme l'évoque un article de presse en 1912 :

«C'était hier à Melun le recensement des nomades qui se trouvent actuellement sur le territoire de Seine-et-Marne. Depuis plusieurs jours, les gendarmes parcourant les routes du département leur avaient assigné comme rendez-vous l'hôtel de la Préfecture de Melun [...]. Sans heurt, joyeusement même, les opérations se poursuivirent de huit heures du matin à trois heures de l'après-midi.

54. Filhol E., Un camp de concentration français..., op. cit.

55. Circulaire du 27 février 1920, CAC 19940494/111, dossier 6270.

56. Cf. Vohl P.-G., Police des ambulants, forains et nomades et des professions connexes, Paris, Charles-Lavauzelle, 1932, pp. 157-168. 
Hommes, femmes, enfants, tous défilèrent devant le photographe et se prêtèrent de bonne grâce aux diverses et minutieuses opérations de l'anthropométrie 57 ».

Les mesures d'identification prévues, qui se déroulent donc plutôt pacifiquement, n'en demeurent pas moins un rite de passage des individus et des familles entières soumis au regard scrutateur et dominateur des forces de l'ordre. Ces opérations nécessitent un matériel spécialisé et un personnel formé aux méthodes d'identification judiciaire. Les mensurations anthropométriques, le signalement judiciaire, la prise des empreintes digitales et la photographie judiciaire sont réalisés dans des laboratoires adaptés ou parfois en plein air, mais généralement dans les commissariats, brigades de gendarmerie ou dans les centres de détention spécialement équipés. Le témoignage d'Harry Söderman éclaire par exemple les conditions dans lesquelles étaient réalisées ces opérations, autour de 1930, dans le laboratoire de police scientifique de Lyon 58 :

" Il y avait là un grand bonhomme barbu, le type même du patriarche, de toute évidence un Gitan. Derrière lui se bousculaient des femmes et une ribambelle d'enfants qui semblaient à première vue innombrables, mais dont le nombre plus tard se révéla comme étant de dix-sept. C'était une tribu au complet [...]. La loi en France impose à tout nomade, Gitan ou autre, vieux ou jeune, mâle ou femelle, de posséder un livret d'identité spécial. Nous avions donc non seulement à photographier toute la bande, à prendre les empreintes digitales, mais encore à bertillonner chacun de ses membres. C'était un travail ingrat, mais trois d'entre nous s'y attelèrent, l'un photographiant et prenant les empreintes, le deuxième établissant les livrets, le troisième traînant les Gitans près de la fenêtre et criant au précédent les chiffres des mensurations et les portraits parlés. À force d'énergie, nous en terminâmes vers midi 59 ».

Les formes aléatoires et imprévues du contrôle étaient susceptibles de conduire, suivant ce récit, à un certain degré d'improvisation, mais les opérations d'identification, présentées ici comme longues et complexes, pouvaient être assurées avec une certaine célérité. Malgré l'absence d'étude précise sur le sujet, il semble bien que les policiers et gendarmes français aient acquis dans

57. «Comment on mesure les nomades », Le Matin, 12 décembre 1912.

58. Harry Söderman (1902-1956), expert en criminalistique, collaborateur d'Edmond Locard à Lyon entre 1926 et 1932, est connu pour son rôle scientifique auprès d'Interpol après 1945. Cf. Larson C., "Harry Soderman of Stockholm: Master Criminologist”, The Journal of Criminal Law, Criminology, and Police Science, n 1, 1952, pp. 95-102 ; Margot P.A., "Harry Söderman. A Great Pan-European Criminalist, 1902-1956”, Problems of Forensic Sciences, 2002, pp. 122-134.

59. Söderman H., Quarante ans de police internationale, trad. J. David, Paris, Presses de la Cité, 1956, pp. 76-77. 
les années 1920 une forme d'expérience, fondée sur la pratique, permettant d'effectuer dans un temps réduit des opérations d'immatriculation sur des groupes relativement importants.

Les lacunes du système de contrôle mis en place se révèlent davantage dans l'impossibilité d'effectuer une surveillance réelle des déplacements effectués par les groupes mobiles de Tsiganes. En effet, l'obligation de présenter le carnet anthropométrique à l'arrivée dans une commune ainsi qu'au départ n'était pas assortie d'une transmission systématique de l'information auprès des départements voisins. Si la procédure d'enregistrement revêtait une fonction importante dans l'exercice du contrôle et si la présentation répétée de ce document d'identité, parfois quotidiennement, devant les différents agents de l'État contribuait à désigner les Tsiganes comme une catégorie de la population particulièrement stigmatisée, la fluidité des déplacements restait très largement une réalité. C'est pour pallier cette défaillance structurelle du contrôle de la mobilité que plusieurs mesures, prises parfois à l'échelle des préfectures, tentent d'apporter une série d'améliorations.

Plusieurs instructions soulignent par la suite la nécessité de signaler sur les carnets, par l'imposition d'un visa, le passage des nomades dans les commissariats ou les brigades de gendarmerie. Afin de suivre les individus à la trace, il est également prévu de conserver pour chaque visa apposé par les autorités, policiers, gendarmes et maires des communes traversées, un feuillet mobile comportant le nom des individus et, le cas échéant, le nom de tous les membres de la famille inscrits sur les carnets anthropométriques, le numéro des carnets visés, des indications de date et de lieu sur l'itinéraire suivi par les individus. Il est alors prévu de classer ces fiches dans un fichier chronologique spécial conservé pour les villes par les commissariats et pour les communes rurales par les brigades de gendarmerie. Un répertoire alphabétique correspondant devait permettre en outre de retrouver le nom des individus recherchés. La création d'un fichier anthropométrique avait été suggérée dès 1913 par le service de l'identité judiciaire de la Préfecture de police, mais cette idée qui reposait sur la réalisation de fiche de mensuration pour chaque nomade enregistré ne fut pas appliquée 60.

En 1920, la réglementation, résultant d'un accord entre la Sûreté générale du ministère de l'Intérieur et la direction de la gendarmerie du ministère de la Guerre, prévoyait l'échange de données entre les commissariats et les brigades de gendarmerie :

60. Note du Contrôle général des services de recherches judiciaires, 22 novembre 1913, AN CAC 19940494/111, dossier 6270. Cette note indique en effet : "Le service de l'identité judicaire a fait remarquer qu'en l'absence de la fiche anthropométrique il ne serait pas possible de découvrir à l'avenir les nomades qui viendraient à prendre un nowvel état civil. De graves inconvénients résulteraient de cet état de choses, le but poursuivi par le législateur ne serait qu'imparfaitement atteint puisque les nomades pourraient impunément continuer à changer de nom ». 
«La communication réciproque des registres et des renseignements qu'ils contiendront sera obligatoire pour les commissaires de police et pour les chefs de brigade de gendarmerie. De même, les commissaires de police devront donner, à charge de réciprocité, les mêmes renseignements aux officiers de la gendarmerie qui les leur demanderaient ${ }^{61}$ ».

Ces précisions traduisent en filigranes les défauts récurrents de la communication entre les différentes forces de police. Certes, la coordination entre police et gendarmerie s'était largement renforcée avant 1914 et les échanges entre les agents existaient dans les faits : la création des brigades mobiles, en 1907, avait en particulier suscité un rapprochement entre les agents des différentes forces et s'appuyait explicitement sur un principe de coopération 62 . Malgré l'amélioration certaine des relations entre police et gendarmerie au lendemain de la Première Guerre mondiale, il semble toutefois que la collaboration restait encore très imparfaite, en particulier dans le contrôle des nomades. En 1920, le souci de garantir désormais la circulation des informations à travers une pratique courante de transmission des feuillets mobiles individuels et collectifs et des registres alphabétiques traduit la volonté d'établir un véritable quadrillage du territoire pour exercer un contrôle, à la fois dans l'espace et dans le temps de la mobilité tsigane en France. L'innovation représentée par la création de fichiers locaux intervient en effet pour renforcer la surveillance de la mobilité associée, dans la note jointe à la circulaire, aux comportements délictueux des Tsiganes :

«Jusqu’à présent, les autorités qui visaient les carnets de nomades ne prenaient pas note du stationnement ou du passage de ces individus. Il en résultait que la poursuite de ceux d'entre eux qui s'étaient rendus coupables d'un crime ou d'un délit donnait lieu à des recherches le plus souvent très laborieuses, et parfois même infructueuses 63 ».

61. Circulaire du ministère de l'Intérieur, 13 novembre 1920 (cf. Décrets annotés. Décret du 10 septembre 1935, avec circulaire du 22 novembre 1935 et instruction du 27 juin 1929 sur l'organisation de la gendarmerie et décret du 20 mai 1903 sur le service de la gendarmerie, Paris, Charles-Lavauzelle, 1939, pp. 320-321) ; circulaire de la direction de la gendarmerie du 8 novembre 1920, relative à l'adoption d'un registre de nomades destiné à faciliter leur surveillance par la gendarmerie, CAC 19940494/111, dossier 6270.

62 . Cf. Berlière J.-M., « La seule police qu'une démocratie puisse avouer ? Retour sur un mythe : Les brigades du Tigre ", in Baruch M.-O. (dir.), Serviteurs de l'État. Une histoire politique de l'administration française, 1875-1945, Paris, La Découverte, 2000, pp. 311-323 ; López L., «Etre mobile : la circulation d'une épithète entre gendarmerie et police (1871-1914) », in Berlière J.-M., Denys C., Kalifa D., Milliot V. (dir.), Métiers de police..., op. cit., pp. 439-451; Brousseaud C., « Les brigades territoriales de gendarmerie et l'exercice de l'enquête judiciaire (1890-1928) », in Farcy J.-C., Kalifa D., Luc J.-N. (dir.), L'enquête judiciaire en Europe an XIXe siècle. Acteurs, imaginaires, pratiques, Paris, Créaphis, 2007, pp. 117-126.

63. Cf. Circulaire de la direction de la gendarmerie, 8 novembre 1920, op. cit. 
La même année, la Préfecture de police établit une équipe spécialisée dans la surveillance des Tsiganes, composée d'une équipe d'inspecteurs de la direction de la police judiciaire. Suivant une circulaire spéciale, les agents de cette "police des nomades » sont chargés de renouveler les contrôles, de vérifier les informations enregistrées précédemment et l'exactitude des données à chaque délivrance de visa sur les carnets individuels et collectifs ${ }^{64}$. Cependant, ces mesures ne semblent pas suffisantes pour limiter le franchissement des frontières car la transmission des feuillets est loin d'être effectuée régulièrement. En 1922, une circulaire de rappel évoque en effet les difficultés rencontrées dans la mise en œuvre du fichier des nomades qui repose essentiellement sur les mairies, chargées d'enregistrer la venue et le départ des Tsiganes. Or, il apparaît que les consignes ne sont pas parvenues dans toutes les mairies et que les formulaires nécessaires n'ont pas été réalisés en nombre suffisant ${ }^{65}$. L'absence de moyens financiers et de personnel pour exercer ce travail administratif supplémentaire apparât aussi parmi les raisons avancées. À la fin des années 1920, comme le conclut une enquête sur la tenue des fichiers des commissariats en France, le fichier des nomades apparât toujours généralement incomplet et ne fait pas l'objet du même soin que les autres fichiers réglementaires ${ }^{6}$.

Dans le contexte d'un durcissement des mesures de contrôle de l'immigration de travail, réglementée par l'imposition de la "carte d'identité des étrangers », créée en 1917, la question soulevée par l'entrée sur le territoire de caravanes tsiganes prend une place de plus en plus centrale ${ }^{67}$. En 1925, une note du ministre de l'Intérieur de l'époque, Camille Chautemps, adressée aux préfets, porte directement sur «le nombre chaque jour plus important de nomades étrangers se présentant aux préfectures et sous-préfectures de certains départements frontières en vue d'obtenir le carnet anthropométrique d'identité 68 ». Le propos central de cette note est de parvenir à empêcher totalement l'entrée de Tsiganes étrangers et d'attirer l'attention sur la question de leur nationalité :

«Il ne vous échappera pas cependant que la présence de ces nomades étrangers sur notre territoire n'est aucunement désirable [...]. Aussi, en vue de remédier à cet état de choses, je vous prie de vouloir bien rappeler à vos collaborateurs les prescriptions aux

64 . Circulaire du Préfet de police, « Police des nomades. Formalités à remplir lors du visa des carnets individuels ou collectifs », 13 décembre 1920, Archives de la Préfecture de police, D/b 200.

65. Circulaires des 8 juin 1922, 29 novembre 1922 et 9 mai 1924, AN CAC 19940494/111, dossier 6288.

66. Cf. l'ensemble des questionnaires réalisés en mai 1928, AN CAC 19940500/12, dossier 138.

67. Cf. About I., «Identifier les étrangers. Genèses d'une police bureaucratique de l'immigration dans la France de l'entre-deux-guerres ", in Noiriel G. (dir.), L'identification des personnes..., op. cit., pp. 125-160.

68. Note du ministre de l'Intérieur aux préfets, « Nomades étrangers », 22 janvier 1925, AN CAC 19890519/5, dossier 1. 
termes [desquelles] les préfets et sous-préfets des départements limitrophes des états étrangers ne devront jamais délivrer de carnet anthropométrique aux nomades venant de ces États s'ils ne sont pas porteurs de pièces authentiques établissant qu'ils possèdent réellement la nationalité française [...]. J'attache la plus grande importance à la stricte exécution de ces instructions dont l'inobservation est de nature à accroître notre population flottante d'éléments nettement indésirables 69 ».

Ces règlements sont suivis rapidement de plusieurs mesures qui interdisent complètement l'entrée sur le territoire national de " nomades étrangers » 70 . Mais il faut attendre une nouvelle phase aiguë de tensions aux frontières pour qu'un accord formel intervienne avec certains pays voisins.

En 1930, une tribu désignée par les autorités françaises comme des nomades yougoslaves, déjà refoulés de France en 1927, se présente à la frontière avec la Belgique. Le ministre de l'Intérieur demande alors à l'ensemble des forces de l'ordre du pays de "vouloir bien prendre les mesures les plus rigoureuses pour [s']opposer à toute intrusion de ces nomades sur notre territoire ${ }^{71} »$. Suivant un article publié en 1932 par Le Matin, cette même famille, guidée par Damon Roussalini, alias Jacob Weiss, aurait été expulsée de France en 1927 vers le Monténégro puis aurait traversé successivement l'Italie, l'Autriche, la Bavière, la Hollande puis la Belgique. En outre, en août 1931, une bande de près de 70 personnes se présente également à la frontière francobelge et provoque un conflit retentissant entre les gendarmes des deux pays. C'est dans ce contexte immédiat que des rencontres ont lieu entre les autorités de Police et de Justice de la France, de la Belgique et du Luxembourg. Les accords signés s'appuient sur le constat des troubles répétés aux frontières dus aux bandes de nomades :

«Ils séjournent sur les confins des trois États ou leur présence a été, jusqu'à l'année dernière, la cause de multiples incidents entre les différents services de police et de gendarmerie chargés du contrôle des frontières 72 ».

\section{Ibid.}

70. Circulaire de la Sûreté générale, «Nomades de nationalité étrangère », 8 novembre 1926 ; Circulaire de la Sûreté générale, "Nomades étrangers ", 26 février 1930, AN CAC 19940494/111, dossier 6288.

71. Cf. Perrigault J., "Les compagnons de la Belle-étoile. La tribu tsigane des Roussalini cauchemar de la police française », Le Matin, 19 juillet 1932. Jean Perrigault est l'auteur d'une grande enquête sur les Tsiganes d'Europe de l'Est et de France, publiée du 10 au 21 juillet 1932. Journaliste et savant, Perrigault est connu pour ses études sur l'Afrique noire éditées dans les années 1930.

72. Circulaire de la Sûreté générale, "Nomades étrangers », 2 juillet 1932, AN CAC 19940494/111, dossier 6288 . 
Afin de remédier à cet état de fait, il est désormais prévu d'organiser les opérations de refoulement sur la base d'un traitement individualisé. Aucune expulsion d'un nomade ne pourra ainsi être effectuée «sans que l'État de destination ait préalablement été avisé et ait accepté de le recevoir »; le terme même de refoulement est dès lors défini comme «non seulement une décision administrative régulièrement prise et notifiée aux intéressés, mais encore [comme] toute mesure tendant à faciliter aux nomades le passage de la frontière 73 ». Ainsi, le passage de la frontière n'est désormais autorisé qu'aux nomades reconnus comme appartenant à la nation d'accueil. Les bases de l'arrangement s'appuient sur des enquêtes de vérification de l'état civil qui doivent confirmer avec certitude la nationalité des individus, une décision s'appliquant au «chef de famille » étant valable pour l'ensemble de sa famille. Cette réglementation doit donc conduire à une fermeture de la frontière et délègue aux autorités la responsabilité d'autoriser ou non le passage sur présentation des documents requis ${ }^{74}$. En somme, la mobilité tsigane transfrontalière est désormais calquée, dans le cadre de cet accord, sur le modèle des migrations transnationales de travail soumises à la délivrance d'un visa sans lequel le passage de frontière devient illégal. Les services de l'État contribuent ainsi au processus consistant à monopoliser « les moyens légitimes de circulation » en imposant aux Tsiganes la nécessité de régulariser leur état civil au regard du droit des nationalités et de posséder une identité civile soumise au principe commun ${ }^{75}$. Il reste toutefois à mesurer la réalité des accords signés en 1931 et à saisir leur rôle dans les transformations de la mobilité tsigane européenne dans les années 1930, lourdement marquée par le nouveau poids de ces mesures limitatives ${ }^{76}$. Mais pour certains observateurs de l'époque, il s'agit bien d'une étape essentielle dans la disparition souhaitée de l'« entité tsigane» :

« Des mesures rigoureuses viennent d'être prises pour rendre à peu près impossible l'entrée en France de nouveaux Tsiganes et Gitanos : ceux qui vivent sur notre territoire sont constamment surveillés. De sorte qu'il ne semble pas téméraire d'affirmer que, dans quelques années, le "nomadisme" aura cessé d'exister en France 77 ».

73. Ibid.

74. Cf. «Accords conclus à Feignies le 13 novembre 1931 et à Luxembourg le 3 mai 1932 au sujet du refoulement des nomades ", AN CAC 19940494/111, dossier 6288.

75. Cf. Torpey J., «Aller et venir : le monopole étatique... », op. cit.; Torpey J., L'invention $d u$ passeport. États, citoyenneté et surveillance, trad. E. Lamothe, Paris, Belin, 2000.

76. L'année suivante, un rapport des brigades mobiles témoigne des tensions suscitées par « une tribu de 56 nomades étrangers qui tentaient de pénétrer en France ». Un fonctionnaire de la Sûreté se rend alors à Bruxelles pour confirmer les termes de l'accord signé précédemment et obtient que « toute la tribu, hommes, femmes, enfants, animaux et matériel » soit accueillie par la gendarmerie belge, cf. « Rapport mensuel sur l'activité de la police mobile », 4 février 1932, AN CAC 19940493/187, dossier 3463.

77 . Perrigault J., «Les compagnons de la Belle-étoile. Bohémiens français », Le Matin, 21 juillet 1932. 
Pour les caravanes habituées à franchir les frontières et traverser ces lignes imaginaires, qui se transforment peu en peu en barrière physique, les conséquences de cette mutation ne sont pas bien connues et mériteraient une enquête approfondie. Le démantèlement d'un réseau séculaire de circulation correspond vraisemblablement à l'affaiblissement des réseaux d'entraide et à l'appauvrissement graduel de communautés déjà soumises à une grande précarité matérielle. De plus, l'aggravation des formes de contrôle et la violence vécue face à la présence récurrente des agents de l'autorité, des outils de l'anthropométrie, des murs des prisons ou des bornes aux frontières suscita sans doute, en retour, une mise en retrait des principes de la négociation, voire un raidissement dans la perception des non-Tsiganes. Au-delà de cette question, qui intéresse la position historique du monde tsigane dans l'histoire européenne, l'amplification du quadrillage des zones frontalières, qui s'accélère dans les années 1930, décide un basculement définitif de leur statut. Sommés de démontrer leur nationalité alors même qu'ils ne sont pas acceptés comme citoyens à part entière, relégués aux marges de la société et confinés dans les limites d'un cadre administratif et policier particulièrement contraignant, ils sont destinés à cet affrontement répété avec les autorités et les populations, à cette pièce rejouée périodiquement en d'autres lieux, avec d'autres textes et d'autres acteurs. Dans ce processus, l'investissement premier des forces de l'ordre fut d'améliorer la transmission des informations pour figer les identités dans le temps et l'espace afin d'anticiper la mobilité et prévenir une migration jugée alors indésirable. Nul doute que la recherche des moyens pour contrer les mobilités tsiganes transnationales joua un rôle central dans l'avènement d'une coopération policière, destinée à cerner les individus à travers un maillage à la fois invisible et massif. 